TRANSACTIONS OF THE

AMERICAN MATHEMATICAL SOCIETY

Volume 358, Number 1, Pages 39-57

S 0002-9947(05)04045-6

Article electronically published on August 25, 2005

\title{
A GENERALIZATION OF EULER'S HYPERGEOMETRIC TRANSFORMATION
}

\author{
ROBERT S. MAIER
}

\begin{abstract}
Euler's transformation formula for the Gauss hypergeometric function ${ }_{2} F_{1}$ is extended to hypergeometric functions of higher order. Unusually, the generalized transformation constrains the hypergeometric function parameters algebraically but not linearly. Its consequences for hypergeometric summation are explored. It has as a corollary a summation formula of Slater. From this formula new one-term evaluations of ${ }_{2} F_{1}(-1)$ and ${ }_{3} F_{2}(1)$ are derived by applying transformations in the Thomae group. Their parameters are also constrained nonlinearly. Several new one-term evaluations of ${ }_{2} F_{1}(-1)$ with linearly constrained parameters are derived as well.
\end{abstract}

\section{INTRODUCTION}

Many hypergeometric identities are known (see Slater [Sla66] and Prudnikov et al. [PBM90, Chapter 7]). Most are summation formulas for hypergeometric series, either infinite or terminating, including binomial coefficient summations Gou72. Recent work has concentrated on developing techniques for verifying asserted or conjectured identities, rather on than deriving new ones. Zeilberger's algorithm and the Wilf-Zeilberger method can verify nearly all classical hypergeometric identities, at least those involving terminating series, and many more besides Koe98, PWZ96. Under the circumstances, maintaining an exhaustive list of such identities has come to be seen as unnecessary. It has even been provocatively asserted that "there is no hypergeometric database," in the sense that the class of hypergeometric identities is for practical purposes open-ended, and that such identities are best proved by algorithmic techniques more sophisticated than database lookup [PWZ96, Chapter 3].

But hypergeometric function transformations, in which the argument is a free variable, are more manageable than general identities. One could reasonably hope to enumerate or otherwise characterize the class, say, of all two-term function transformations. In fact the two-term transformations relating the Gauss hypergeometric function ${ }_{2} F_{1}(x)$ to ${ }_{2} F_{1}(R x)$, where $R$ is a rational map of the Riemann sphere to itself, are now fully classified. Besides the celebrated transformation of Euler,

$$
{ }_{2} F_{1}\left(\begin{array}{c|c}
a, b & x \\
c & x
\end{array}\right)=(1-x)^{c-a-b}{ }_{2} F_{1}\left(\begin{array}{c|c}
c-a, c-b & x \\
c & x
\end{array}\right),
$$

in which $R(x)=x$, and Pfaff's transformation, in which $R(x)=x /(x-1)$, there are transformations of larger mapping degree $(\operatorname{deg} R>1)$, which were classified by

Received by the editors April 11, 2003.

2000 Mathematics Subject Classification. Primary 33C20; Secondary 33C05, 34Mxx.

This work was partially supported by NSF grant PHY-0099484.

(C)2005 American Mathematical Society 39

Reverts to public domain 28 years from publication 
Goursat Gou81. The best known are the quadratic ones, which were originally worked out by Gauss and Kummer, and proved concisely by Riemann AAR99, $\S 3.9]$. Recently, Goursat's classification was completed by an enumeration of the transformations of ${ }_{2} F_{1}$ with no free parameter, most of which have quite large degree Vid04. Several of the quadratic and cubic transformations of ${ }_{2} F_{1}$ have analogues on the ${ }_{3} F_{2}$ level, discovered by Whipple and Bailey respectively [Ask94. 'Companion' transformations relate ${ }_{3} F_{2}$ to ${ }_{4} F_{3}$ GS82. But no clear analogues on levels above ${ }_{2} F_{1}$ of the remaining ${ }_{2} F_{1}$ transformations, in particular of the degree-1 transformations of Euler and Pfaff, have previously been found.

We report here on a recent discovery: Euler's transformation (1.1) has an analogue on all higher levels. That this has not been noticed before may be due to the fact that in the generalized transformation, the hypergeometric parameters are constrained algebraically, not linearly. This is very unusual. In the compilation of Prudnikov et al. [PBM90, Chapter 7], no hypergeometric identity with parameters constrained in this way is listed. The only published hypergeometric function transformation with nonlinearly constrained parameters that we have been able to uncover is a quadratic one connecting ${ }_{3} F_{2}$ and ${ }_{5} F_{4}$ [Nib53, which seems unrelated.

The generalized Euler transformation came close to being discovered in the 1950s by Slater, who obtained a formula for ${ }_{r+1} F_{r}(1)$ that applies when its parameter vector is restricted to a certain algebraic variety Sla55. Several closely related summation formulas with nonlinear parametric restrictions had been obtained much earlier by Searle Sea09. Searle's and Slater's formulas have not attracted the attention they deserve, though Slater's was later reproduced in Sla66, § 2.6.1]. More recently, a few additional expressions for hypergeometric sums with algebraically constrained parameters have been published. These include a formula for a terminating ${ }_{4} F_{3}(1)$ Luk69, §3.13], a related one for a terminating ${ }_{3} F_{2}(1)$ GS82, Eq. (1.9)], an evaluation of a nonterminating ${ }_{4} F_{3}(1)$ [Gos76], and an exotic evaluation of a nonterminating ${ }_{2} F_{1}(-1)$ Vid02, $\left.\S 4\right]$.

This article is organized as follows. The generalized Euler transformation appears as Theorem 2.1. A combinatorial proof is given, and Slater's summation formula and several additional hypergeometric identities are derived as corollaries. In Section 3 the implications for ${ }_{3} F_{2}(1)$ are examined. Using its $S_{5}$ symmetry, a set of three related ${ }_{3} F_{2}(1)$ evaluations is derived, each with nonlinear parametric constraints and three free parameters. The extent to which they overlap the standard identities of Dixon, Watson, Whipple, and Pfaff-Saalschütz is determined. In Section 4 these new identities are employed to generate new evaluations of ${ }_{2} F_{1}(-1)$, including several exotic ones with nonlinearly constrained parameters.

\section{Key RESULts}

The following standard notation will be used. For any integer $r \geqslant 1$ and parameter vector $(\alpha ; \beta)=\left(\alpha_{1}, \ldots, \alpha_{r+1} ; \beta_{1}, \ldots, \beta_{r}\right) \in \mathbb{C}^{r+1} \times \mathbb{C}^{r}$ in which no $\beta_{i}$ is a nonpositive integer, the generalized hypergeometric series is

$$
{ }_{r+1} F_{r}\left(\begin{array}{c}
\alpha_{1}, \ldots, \alpha_{r+1} \\
\beta_{1}, \ldots, \beta_{r}
\end{array} \mid x\right)=\sum_{k=0}^{\infty} \frac{\left(\alpha_{1}\right)_{k} \cdots\left(\alpha_{r+1}\right)_{k}}{\left(\beta_{1}\right)_{k} \cdots\left(\beta_{r}\right)_{k}} \frac{x^{k}}{k !}
$$

where $(\alpha)_{k}$ signifies the rising factorial $\alpha(\alpha+1) \cdots(\alpha+k-1)$, with $(\alpha)_{0}=1$. If $x$ is omitted, $x=1$ is understood. The series converges absolutely on $|x|<1$, and if the parametric excess $s=\sum_{i=1}^{r} \beta_{i}-\sum_{i=1}^{r+1} \alpha_{i}$ has positive real part, it 
converges at $x=1$. (For convergence at $x=-1$, Re $s>-1$ suffices.) The series can be continued uniquely from the unit disk to the cut Riemann sphere $\mathbb{C P}^{1} \backslash[1, \infty]$. Any hypergeometric transformation formula, when stated without restriction on $x$, should be taken as holding in a neighborhood of $x=0$, namely the largest neighborhood of $x=0$ in $\mathbb{C P}^{1} \backslash[1, \infty]$ to which both sides can be continued.

A generalized hypergeometric series or function is said to be $s$-balanced if the parametric excess equals $s$. It is well-poised if the parameters $\left\{\alpha_{i}\right\},\left\{\beta_{i}\right\}$ can be separately permuted so that $\alpha_{1}+1=\alpha_{2}+\beta_{1}=\cdots=\alpha_{r+1}+\beta_{r}$.

The generalized Euler transformation formula will now be stated and proved.

Definition. For each $r \geqslant 1$, the algebraic variety $\mathcal{U}_{r} \subset \mathbb{C}^{r+1} \times \mathbb{C}^{r}$, which is $(r+1)$ dimensional, comprises all $(\alpha ; \beta)$ for which the $r$ equations

$$
\left\{\begin{aligned}
\sum_{1 \leqslant i \leqslant r+1} \alpha_{i} & =\sum_{1 \leqslant i \leqslant r} \beta_{i} \\
\sum_{1 \leqslant i<j \leqslant r+1} \alpha_{i} \alpha_{j} & =\sum_{1 \leqslant i<j \leqslant r} \beta_{i} \beta_{j}, \\
\sum_{1 \leqslant i<j<k \leqslant r+1} \alpha_{i} \alpha_{j} \alpha_{k} & =\sum_{1 \leqslant i<j<k \leqslant r} \beta_{i} \beta_{j} \beta_{k}, \\
& \vdots
\end{aligned}\right.
$$

are satisfied. In the $k^{\prime}$ th equation, the left and right sides are the $k$ 'th elementary symmetric polynomial in $\alpha_{1}, \ldots, \alpha_{r+1}$ and in $\beta_{1}, \ldots, \beta_{r}$, respectively.

Theorem 2.1. For all $r \geqslant 1$ and $(\alpha ; \beta) \in \mathcal{U}_{r}$ for which no $\beta_{i}+1$ is a nonpositive integer,

$$
{ }_{r+1} F_{r}\left(\begin{array}{c}
\alpha_{1}, \ldots, \alpha_{r+1} \\
\beta_{1}+1, \ldots, \beta_{r}+1
\end{array} \mid x\right)=(1-x)_{r+1} F_{r}\left(\begin{array}{c}
\alpha_{1}+1, \ldots, \alpha_{r+1}+1 \\
\beta_{1}+1, \ldots, \beta_{r}+1
\end{array} \mid x\right) .
$$

Remark. By the condition $(\alpha ; \beta) \in \mathcal{U}_{r}$, the series on the left and right sides are respectively $r$-balanced and (-1)-balanced. If $r=1$, this reduces to the 1 -balanced case of Euler's transformation, i.e., the case $c-a-b=1$ of (1.1).

Proof of Theorem 2.1. By the series representation (2.1), the theorem will follow if for all $k \geqslant 1$,

$$
\begin{aligned}
& \frac{\left(\alpha_{1}\right)_{k} \cdots\left(\alpha_{r+1}\right)_{k}}{\left(\beta_{1}+1\right)_{k}} \cdots\left(\beta_{r}+1\right)_{k} k ! \\
& =\frac{\left(\alpha_{1}+1\right)_{k} \cdots\left(\alpha_{r+1}+1\right)_{k}}{\left(\beta_{1}+1\right)_{k} \cdots\left(\beta_{r}+1\right)_{k} k !}-\frac{\left(\alpha_{1}+1\right)_{k-1} \cdots\left(\alpha_{r+1}+1\right)_{k-1}}{\left(\beta_{1}+1\right)_{k-1} \cdots\left(\beta_{r}+1\right)_{k-1}(k-1) !} .
\end{aligned}
$$

By examination, this equation can be obtained from

$$
\alpha_{1} \cdots \alpha_{r+1}=\left(\alpha_{1}+k\right) \cdots\left(\alpha_{r+1}+k\right)-\left(\beta_{1}+k\right) \cdots\left(\beta_{r}+k\right) k
$$

by multiplying by the product $\left(\alpha_{1}+1\right)_{k-1} \cdots\left(\alpha_{r+1}+1\right)_{k-1}$ and dividing by the product $\left(\beta_{1}+1\right)_{k} \cdots\left(\beta_{r}+1\right)_{k} k$ !. The left side of (2.2) is independent of $k$, and the right side is a polynomial in $k$ of degree $r$. For $n=1, \ldots, r$, the coefficient of $k^{n}$ is proportional to the sum of all monomials $\alpha_{i_{1}} \cdots \alpha_{i_{r+1-n}}$ minus the sum of all monomials $\beta_{j_{1}} \cdots \beta_{j_{r+1-n}}$. But since $(\alpha ; \beta) \in \mathcal{U}_{r}$, each coefficient is zero. So the right side of (2.2) reduces to a constant, namely $\alpha_{1} \cdots \alpha_{r+1}$, and is the same as the left side. The theorem is proved.

Theorem 2.1 can be viewed as belonging to the theory of contiguous function relations, which (for ${ }_{2} F_{1}$ ) dates back to Gauss. Recall that if a parameter of ${ }_{r+1} F_{r}$ is displaced by \pm 1 , the resulting function is said to be contiguous to the original. Gauss showed that ${ }_{2} F_{1}(a, b ; c ; x)$ and any two of its contiguous series are 
connected by a homogeneous linear relation with coefficients linear in $x$ and polynomial in $(a, b ; c)$. In fact by constructing chains of contiguous functions, he showed that any series of the form ${ }_{2} F_{1}(a+l, b+m ; c+n ; x)$, where $(l, m ; n) \in \mathbb{Z}^{2} \times \mathbb{Z}$, is connected to ${ }_{2} F_{1}(a, b ; c ; x)$ and any one of its contiguous series by a three-term homogeneous linear relation, the coefficients of which are polynomial in $x$ and $(a, b ; c)$. By imposing parametric constraints, i.e., confining $(a, b ; c)$ to an algebraic variety, it is sometimes possible to obtain two-term relations, as well.

The two-term relation of Theorem 2.1 is clearly related to this classical theory, but is much stronger in that it holds for all $r \geqslant 1$. Contiguous function relations for arbitrary ${ }_{r+1} F_{r}$ (and indeed for arbitrary ${ }_{p} F_{q}$ ) were worked out by Rainville Rai45. There is a fundamental set of $3 r+2$ such relations, with up to $r+2$ terms, from which all others follow by linearity. But the theory of those relations is complicated, since Gauss's construction does not extend to $r>1$. In particular, no two-term relation for general $r$ was previously known. Theorem 2.1 is therefore surprising.

When applied to Euler's transformation, the technique of equating coefficients produces the Pfaff-Saalschütz formula, which sums any terminating 1-balanced ${ }_{3} F_{2}(1)$ AAR99, $\left.\S 2.2\right]$. Applied to Theorem 2.1, it produces the following.

Theorem 2.2. For all $r \geqslant 1$ and $(\alpha ; \beta) \in \mathcal{U}_{r}$ for which no $\beta_{i}+1$ is a nonpositive integer,

$$
\sum_{j=0}^{n} \frac{\left(\alpha_{1}\right)_{j} \cdots\left(\alpha_{r+1}\right)_{j}}{\left(\beta_{1}+1\right)_{j} \cdots\left(\beta_{r}+1\right)_{j} j !}=\frac{\left(\alpha_{1}+1\right)_{n} \cdots\left(\alpha_{r+1}+1\right)_{n}}{\left(\beta_{1}+1\right)_{n} \cdots\left(\beta_{r}+1\right)_{n} n !}
$$

for every integer $n \geqslant 0$. In consequence, for all $r \geqslant 1$ and $(\alpha ; \beta) \in \mathcal{U}_{r}$ for which $\alpha_{1}$ equals $-n$, a nonpositive integer, and for which no $\beta_{i}+1$ is a nonpositive integer,

$$
{ }_{r+1} F_{r}\left(\begin{array}{c}
-n, \alpha_{2}, \ldots, \alpha_{r+1} \\
\beta_{1}+1, \ldots, \beta_{r}+1
\end{array}\right)=\frac{\left(\alpha_{1}+1\right)_{n} \cdots\left(\alpha_{r+1}+1\right)_{n}}{\left(\beta_{1}+1\right)_{n} \cdots\left(\beta_{r}+1\right)_{n} n !} .
$$

Proof. To obtain (2.3) from Theorem 2.1. multiply both sides by $(1-x)^{-1}$ and equate the coefficients of $x^{n}$ on the two sides.

The formula (2.4) sums a class of terminating $r$-balanced ${ }_{r+1} F_{r}(1)$ series, in which the parameters are required to satisfy $r-1$ nonlinear conditions. It is worth considering the relation between (2.4) and other summation formulas. Any terminating 2-balanced ${ }_{3} F_{2}(1)$ can be summed by the Sheppard-Anderson formula Roy87, so the $r=2$ case of (2.4) is a special case of a more general known result. When $r>2$, the situation is quite different, since few closed-form summations of terminating $r$-balanced ${ }_{r+1} F_{r}(1)$ 's with $r>2$ are known. Certain terminating 4-balanced ${ }_{5} F_{4}(1)$ 's [Sta98, $\S 6$ ] and terminating 6-balanced ${ }_{7} F_{6}(1)$ 's [Ask89] can be evaluated in closed form, though explicit formulas have not been published. It would be interesting to determine the extent to which (2.4) overlaps those closedform evaluations. For larger values of $r,(2.4)$ is in a league by itself.

The following theorem is the extension of (2.4) to nonterminating series.

Theorem 2.3. For all $r \geqslant 1$ and $(\alpha ; \beta) \in \mathcal{U}_{r}$ for which no $\beta_{i}+1$ is a nonpositive integer,

$$
{ }_{r+1} F_{r}\left(\begin{array}{c}
\alpha_{1}, \ldots, \alpha_{r+1} \\
\beta_{1}+1, \ldots, \beta_{r}+1
\end{array}\right)=\Gamma\left[\begin{array}{c}
\beta_{1}+1, \ldots, \beta_{r}+1 \\
\alpha_{1}+1, \ldots, \alpha_{r+1}+1
\end{array}\right]
$$


Remark. This introduces a useful notation. The right side is a gamma quotient of the sort that appears in many hypergeometric summations. It would be written in full as $\Gamma\left(\beta_{1}+1\right) \cdots \Gamma\left(\beta_{r}+1\right) / \Gamma\left(\alpha_{1}+1\right) \cdots \Gamma\left(\alpha_{r+1}+1\right)$. Any gamma quotient with a denominator argument equal to a nonpositive integer is interpreted as zero.

Proof of Theorem 2.3. Take $n \rightarrow \infty$ in (2.3), using the $p, q=r+1, r$ case of

$$
\frac{\left(\alpha_{1}+1\right)_{n} \cdots\left(\alpha_{p}+1\right)_{n}}{\left(\beta_{1}+1\right)_{n} \cdots\left(\beta_{q}+1\right)_{n} n !} \sim \frac{\Gamma\left(\beta_{1}+1\right) \cdots \Gamma\left(\beta_{q}+1\right)}{\Gamma\left(\alpha_{1}+1\right) \cdots \Gamma\left(\alpha_{p}+1\right)} n^{\sum_{i=1}^{p}\left(\alpha_{i}+1\right)-\sum_{i=1}^{q}\left(\beta_{i}+1\right)-1},
$$

which is well-known $n \rightarrow \infty$ asymptotic behavior [AAR99, $\S 2.1$ ].

Theorems 2.2 and 2.3 are precisely the summation results of Slater. The best reference for her original derivation is the note [Sla55], since the version in [Sla66, $\S 2.6 .1]$ contains several unfortunate transcription errors. In particular, the quantity $a+b+c-d$ in (2.6.1.10)-(2.6.1.11) should be read as $2+a+b+c-d$. It should be mentioned that much earlier, Searle [Sea09] proved a related theorem on partial sums of series of hypergeometric type, from which Slater's results can be deduced as corollaries. (The author is indebted to George Andrews for this reference.)

For every integer $m \geqslant 0$, there is a similar summation formula for a class of $(r+m)$-balanced ${ }_{r+1} F_{r}(1)$ series, with the parameters required to satisfy $r-1$ nonlinear conditions. This result is best framed in terms of an $(r+1)$-dimensional algebraic variety $\mathcal{U}_{r}^{m} \subset \mathbb{C}^{r+1} \times \mathbb{C}^{r}$, indexed by $m$, with $\mathcal{U}_{r}^{0}$ equalling $\mathcal{U}_{r}$. The $r$ polynomial equations defining $\mathfrak{U}_{r}^{m}$ become unwieldy when $m$ is large, so the following exposition of the $m=1$ case should suffice.

If $(\alpha ; \beta) \in \mathbb{C}^{r+1} \times \mathbb{C}^{r}$, let $S_{l}(\alpha)$, resp. $S_{l}(\beta)$, denote the sum of the $\left(\begin{array}{c}r+1 \\ l\end{array}\right)$ monomials of the form $\alpha_{i_{1}} \cdots \alpha_{i_{l}}$, resp. the $\left(\begin{array}{l}r \\ l\end{array}\right)$ monomials of the form $\beta_{j_{1}} \cdots \beta_{j_{l}}$, with $S_{0}(\alpha)=S_{0}(\beta)=1$ by convention. $\mathfrak{U}_{r}^{0}=\mathcal{U}_{r}$ is the common solution set of the equations $S_{l}(\alpha)-S_{l}(\beta)=0, l=1, \ldots, r$. Let $\mathcal{U}_{r}^{1} \subset \mathbb{C}^{r+1} \times \mathbb{C}^{r}$ be the common solution set of the equations

$$
\begin{aligned}
& {\left[S_{l-1}(\alpha)-S_{l-1}(\beta)\right]\left[S_{r+1}(\alpha)+S_{r}(\beta)\right]} \\
& \quad+\left[S_{l}(\alpha)-S_{l}(\beta)+S_{l-1}(\beta)\right]\left[-S_{r}(\alpha)+S_{r}(\beta)\right]=0,
\end{aligned}
$$

$l=1, \ldots, r$. It follows from the $l=1$ equation that $(\alpha ; \beta) \in \mathcal{U}_{r}^{1}$ only if the series ${ }_{r+1} F_{r}\left(\alpha_{1}, \ldots, \alpha_{r+1} ; \beta_{1}+1, \ldots, \beta_{r}+1 ; 1\right)$ is $(r+1)$-balanced, unless $S_{r}(\alpha)=S_{r}(\beta)$.

Theorem 2.4. For all $r \geqslant 1$ and $(\alpha ; \beta) \in \mathcal{U}_{r}^{1}$ for which no $\beta_{i}+1$ is a nonpositive integer and for which $S_{r+1}(\alpha)+S_{r}(\beta) \neq 0$,

$$
{ }_{r+1} F_{r}\left(\begin{array}{c}
\alpha_{1}, \ldots, \alpha_{r+1} \\
\beta_{1}+1, \ldots, \beta_{r}+1
\end{array}\right)=\left[\frac{-S_{r}(\alpha)+S_{r}(\beta)}{S_{r+1}(\alpha)+S_{r}(\beta)}\right] \Gamma\left[\begin{array}{c}
\beta_{1}+1, \ldots, \beta_{r}+1 \\
\alpha_{1}+1, \ldots, \alpha_{r+1}+1
\end{array}\right] .
$$

Proof. Trivially,

$$
{ }_{r+1} F_{r}\left(\begin{array}{c}
\alpha_{1}, \ldots, \alpha_{r+1} \\
\beta_{1}+1, \ldots, \beta_{r}+1
\end{array}\right)={ }_{r+2} F_{r+1}\left(\begin{array}{c}
\alpha_{1}, \ldots, \alpha_{r+1}, \gamma \\
\beta_{1}+1, \ldots, \beta_{r}+1, \gamma
\end{array}\right) .
$$

If the conditions $S_{l}\left(\alpha_{1}, \ldots, \alpha_{r+1}, \gamma\right)-S_{l}\left(\beta_{1}, \ldots, \beta_{r}, \gamma\right)=0, l=1, \ldots, r+1$, are satisfied, then the right-hand series can be summed by Theorem 2.3. It follows from the condition labeled by $l=r+1$ that for this to occur, $\gamma$ must equal $\left[S_{r+1}(\alpha)+S_{r}(\beta)\right] /\left[-S_{r}(\alpha)+S_{r}(\beta)\right]$. Eliminating $\gamma$ from the remaining conditions yields (2.5). The prefactor in the theorem is simply $\Gamma(\gamma) / \Gamma(\gamma+1)=1 / \gamma$. 
So a class of $(r+1)$-balanced ${ }_{r+1} F_{r}(1)$ 's with nonlinearly constrained parameters can be summed in closed form. This is the $m=1$ case of the result mentioned above. The extension to $m>1$ is algebraically nontrivial. For $m=2$, one can write

$$
{ }_{r+1} F_{r}\left(\begin{array}{c}
\alpha_{1}, \ldots, \alpha_{r+1} \\
\beta_{1}+1, \ldots, \beta_{r}+1
\end{array}\right)={ }_{r+3} F_{r+2}\left(\begin{array}{c}
\alpha_{1}, \ldots, \alpha_{r+1}, \gamma, \delta \\
\beta_{1}+1, \ldots, \beta_{r}+1, \gamma, \delta
\end{array}\right),
$$

and solve for the $\gamma, \delta$ for which the right-hand side can be evaluated by Theorem 2.3 . Each of $\gamma, \delta$ will be an irrational algebraic function of $(\alpha ; \beta)$, and the same will be true of the prefactor multiplying the gamma quotient. In fact, this prefactor will be an irrational algebraic function of $(\alpha ; \beta)$ for all $m>1$. So the statement of the extension of Theorem 2.4 to $m>1,(\alpha ; \beta) \in \mathcal{U}_{r}^{m}$, is rather complicated.

Theorems 2.2 2.4 followed from Theorem 2.1, the generalized Euler transformation, by the method of equating coefficients. The beta integral method, which is a more sophisticated way of generating hypergeometric summation formulas from transformation formulas, can also be used. Krattenthaler and Srinivasa Rao KS03. have applied this method to the known transformations of ${ }_{r+1} F_{r}(x)$ to ${ }_{r+1} F_{r}(R x)$. Applying it to Theorem 2.1 yields the following remarkable identity.

Theorem 2.5. For all $r \geqslant 1$ and $(\alpha ; \beta) \in \mathcal{U}_{r}$ for which no $\beta_{i}+1$ is a nonpositive integer,

$$
\begin{aligned}
& { }_{r+2} F_{r+1}\left(\begin{array}{c}
\alpha_{1}, \ldots, \alpha_{r+1}, A \\
\beta_{1}+1, \ldots, \beta_{r}+1, B
\end{array}\right) \\
& \quad=\left(\frac{B-A}{B}\right)_{r+2} F_{r+1}\left(\begin{array}{c}
\alpha_{1}+1, \ldots, \alpha_{r+1}+1, A \\
\beta_{1}+1, \ldots, \beta_{r}+1, B+1
\end{array}\right),
\end{aligned}
$$

for all $A, B \in \mathbb{C}$, provided $\operatorname{Re}(B-A)>0$ and $B$ is not a nonpositive integer.

Remark. Since $(\alpha ; \beta) \in \mathcal{U}_{r}$, the two series are respectively $(B-A+r)$-balanced and $(B-A)$-balanced. If $r=1$ there are no nonlinear constraints, and this identity reduces to a special case of one of Thomae's transformations of ${ }_{3} F_{2}(1)$ (see below).

Proof of Theorem 2.5. Multiply both sides of Theorem 2.1 by $x^{A-1}(1-x)^{B-A-1}$, and integrate from $x=0$ to $x=1$. To evaluate each side, interchange integration and summation, and use the fact that the beta integral $\int_{0}^{1} x^{\mu-1}(1-x)^{\nu-1} d x$ equals $\Gamma(\mu) \Gamma(\nu) / \Gamma(\mu+\nu)$. Finally, convert each side back to hypergeometric notation.

\section{3. ${ }_{3} F_{2}(1)$ SUmmations}

If $\operatorname{Re}(c-a-b)>0$ so that ${ }_{2} F_{1}(a, b ; c ; 1)$ is convergent, its sum can be evaluated as the gamma quotient $\Gamma(c) \Gamma(c-a-b) / \Gamma(c-a) \Gamma(c-b)$ by Gauss's theorem. There is no simple analogue of this fact for ${ }_{r+1} F_{r}(1), r>1$. Characterizing the hypergeometric series that are summable in finite terms is an unsolved problem.

In this section the relation between the $r=2$ case of Slater's formula for ${ }_{r+1} F_{r}(1)$ and several 3-parameter summations with linear parametric restrictions is investigated, and two additional 3-parameter ${ }_{3} F_{2}(1)$ summations with nonlinear parametric restrictions are derived (see Theorem 3.4). Underlying this section is the following question about the level $r=2$, which at present is too hard to answer.

Which algebraic varieties $\mathcal{V} \subset \mathbb{C}^{3} \times \mathbb{C}^{2}$ have the gamma quotient summation property, in the sense that for all parameter vectors $(\alpha ; \beta) \in \mathcal{V}$, the hypergeometric series ${ }_{3} F_{2}\left(\alpha_{1}, \alpha_{2}, \alpha_{3} ; \beta_{1}, \beta_{2} ; 1\right)$ sums to a gamma quotient, the arguments of which 
are affine functions (with rational coefficients) of $\alpha_{1}, \alpha_{2}, \alpha_{3} ; \beta_{1}, \beta_{2}$ ? This is subject of course to $\operatorname{Re}\left(\beta_{1}+\beta_{2}-\alpha_{1}-\alpha_{2}-\alpha_{3}\right)>0$, with no $\beta_{i}$ a nonpositive integer.

A similar question could be posed about any level $r>1$, but some partial results on $r=2$ are available. Wimp Wim83 and Zeilberger [Zei92 proved that $\mathbb{C}^{3} \times \mathbb{C}^{2}$ itself does not have the gamma quotient summation property. For a recent discussion, including remarks on higher $r$, see Wim98. In Wimp's proof, a potential obstruction is associated with any line of the form $\left(\alpha_{1}, \alpha_{2}, \alpha_{3} ; \beta_{1}, \beta_{2}\right)=$ $(a, b, c ; d, 2 c)+\xi(1,1,1 ; 1,2), \xi \in \mathbb{C}$. In Zeilberger's proof a similar role is played by the line $\left(\alpha_{1}, \alpha_{2}, \alpha_{3} ; \beta_{1}, \beta_{2}\right)=(0,0,1 ; 1,1)+\xi(1,1,-1 ; 0,0), \xi \in \mathbb{C}$. No variety $\mathcal{V}$ with such a line as a subvariety can have the property.

However, there are many varieties $\mathcal{V} \subset \mathbb{C}^{3} \times \mathbb{C}^{2}$ that permit the evaluation of ${ }_{3} F_{2}(1)$ as a single gamma quotient. If an upper and a lower parameter are equal, they may be 'cancelled,' reducing the ${ }_{3} F_{2}(1)$ to a ${ }_{2} F_{1}(1)$. This shows the existence of $3 \cdot 2=6$ hyperplanes with the property. Less trivially, each of the classical summation formulas of Dixon, Watson, and Whipple applies when $(\alpha ; \beta)$ is restricted to a certain 3-dimensional affine subspace (a '3-plane') [Sla66, § 2.3]. Dixon's formula for the sum of a well-poised ${ }_{3} F_{2}(1)$ is

$$
{ }_{3} F_{2}\left(\begin{array}{c}
a, b, c \\
1+a-b, 1+a-c
\end{array}\right)=\Gamma\left[\begin{array}{c}
1+\frac{1}{2} a, 1+\frac{1}{2} a-b-c, 1+a-b, 1+a-c \\
1+a, 1+a-b-c, 1+\frac{1}{2} a-b, 1+\frac{1}{2} a-c
\end{array}\right]
$$

and Watson's and Whipple's formulas are respectively

$$
\begin{gathered}
{ }_{3} F_{2}\left(\begin{array}{c}
a, b, c \\
\frac{1}{2}+\frac{1}{2} a+\frac{1}{2} b, 2 c
\end{array}\right)=\Gamma\left[\begin{array}{c}
\frac{1}{2}, \frac{1}{2}+c, \frac{1}{2}+\frac{1}{2} a+\frac{1}{2} b, \frac{1}{2}-\frac{1}{2} a-\frac{1}{2} b+c \\
\frac{1}{2}+\frac{1}{2} a, \frac{1}{2}+\frac{1}{2} b, \frac{1}{2}-\frac{1}{2} a+c, \frac{1}{2}-\frac{1}{2} b+c
\end{array}\right] \\
{ }_{3} F_{2}\left(\begin{array}{c}
a, 1-a, c \\
e, 1+2 c-e
\end{array}\right)=\Gamma\left[\begin{array}{c}
e, 1+2 c-e, \frac{1}{2}+\frac{1}{2} a+\frac{1}{2} e, \frac{1}{2}-\frac{1}{2} a+c-\frac{1}{2} e \\
a+e, \frac{1}{2}+\frac{1}{2} a+c-\frac{1}{2} e, \frac{1}{2}-\frac{1}{2} a+\frac{1}{2} e, 1-a+2 c-e
\end{array}\right] .
\end{gathered}
$$

The parametric excess of each ${ }_{3} F_{2}(1)$ is assumed to have positive real part, with no lower parameter equal to a nonpositive integer. The latter two formulas are often given in alternative forms, which follow from the duplication formula $\Gamma(2 z)=$ $(2 \pi)^{-1 / 2} 2^{2 z-1 / 2} \Gamma(z) \Gamma(z+1 / 2)$.

By Theorem 2.3 the variety comprising all $\left(\alpha_{1}, \alpha_{2}, \alpha_{3} ; \beta_{1}+1, \beta_{2}+1\right)$, where $(\alpha ; \beta) \in \mathcal{U}_{2}$, also has the gamma quotient summation property. In the absence of a list of subvarieties of $\mathbb{C}^{3} \times \mathbb{C}^{2}$ with this property, it is natural to inquire as to the relation between this 3-dimensional 'Slater variety,' which is not a 3-plane, and the better-known 3-planes of Dixon, Watson, and Whipple. Does it play some role, perhaps, in bridging between them?

What it means for two varieties to have a high degree of overlap is clarified by the following standard facts from algebraic geometry. An algebraic variety in the parameter space $\mathbb{C}^{r+1} \times \mathbb{C}^{r}$ is the common zero-set of a collection of polynomials in $(\alpha ; \beta)$. Any irreducible variety (one with a single component) has a well-defined dimension in the sense of algebraic geometry, and the complement of its set of singular points, if any, is a Riemann surface of that dimension. If $\mathcal{X}, y$ are varieties with dimensions $m, n$ respectively, with $m+n \geqslant 2 r+1$, then each component of the intersection $x \cap y$, if it is nonempty, will have dimension greater than or equal to $m+n-(2 r+1)$. If "greater than or equal to" cannot be replaced by "equal to", then $\mathcal{X}, y$ are said to intersect improperly. Proper intersections are generic; improper ones are not, and are more delicate.

Suppose $x, y$ are 3 -dimensional varieties in $\mathbb{C}^{3} \times \mathbb{C}^{2}$ with the gamma quotient summation property. If $x \cap y$ is nonempty, it will have dimension unity and be an 
algebraic curve (if the intersection is proper), or contain a component of dimension greater than unity (if it is improper). The latter would signal the existence of a connection, not necessarily obvious, between the corresponding summation formulas, since it would indicate an unexpected amount of overlap.

It would seem easy to examine the parametric constraints of Slater's formula and Dixon's formula, say, to determine whether when taken together, they result in there being one free parameter or two. But a full examination of the extent of overlap between two summation formulas must take into account the symmetry group of ${ }_{3} F_{2}(1)$ evaluations, of which the following is a description.

There is a trivial symmetry group isomorphic to $S_{3} \times S_{2}$, which arises from separate permutations of upper and lower parameters. So, associated to any summation formula are up to $3 ! 2 !=12$ trivially equivalent formulas. It has long been known that there is a larger symmetry group $\mathfrak{T} \subset G L(5, \mathbb{C})$, generated by the permutations and by certain transformations developed by Thomae [Tho79], which linearly 'mix up' the two sorts of parameters. As Thomae and others showed Har23, the extended group $\mathfrak{T}$, which may be called the Thomae group, is isomorphic to $S_{5}$. It can be realized very concretely [BLS87]. Let

$$
{ }_{3} E_{2}\left(\begin{array}{c}
x, y, z \\
u, v
\end{array}\right)=\frac{1}{\Gamma(d) \Gamma(e) \Gamma(s)}{ }_{3} F_{2}\left(\begin{array}{c}
a, b, c \\
d, e
\end{array}\right),
$$

where $(a, b, c ; d, e)=(x, y, z ; u, v) A$, with the $5 \times 5$ matrix $A$ given by

$$
A=\left(\begin{array}{rrrrr}
1 & 0 & 0 & 1 & 1 \\
0 & 1 & 0 & 1 & 1 \\
0 & 0 & 1 & 1 & 1 \\
1 & 1 & 1 & 2 & 1 \\
1 & 1 & 1 & 1 & 2
\end{array}\right), \quad 3 A^{-1}=\left(\begin{array}{rrrrr}
1 & -2 & -2 & 1 & 1 \\
-2 & 1 & -2 & 1 & 1 \\
-2 & -2 & 1 & 1 & 1 \\
1 & 1 & 1 & 1 & -2 \\
1 & 1 & 1 & -2 & 1
\end{array}\right)
$$

so that separate permutations of $x, y, z$ and $u, v$ correspond to those of $a, b, c$ and $d, e$. This defines ${ }_{3} E_{2}(x, y, z ; u, v)$ at all $(x, y, z ; u, v)$ for which the corresponding ${ }_{3} F_{2}(a, b, c ; d, e ; 1)$ is convergent. The statement of Thomae-covariance is that ${ }_{3} E_{2}$ extends by analytic continuation to a function that is invariant under all 5 ! permutations of its parameters.

Since there are $5 ! / 3 ! 2 !=10$ cosets of $S_{3} \times S_{2}$ in $S_{5},{ }_{3} F_{2}(a, b, c ; d, e ; 1)$ can be written in 10 distinct ways, each of the form $G_{3} F_{2}\left(a^{\prime}, b^{\prime}, c^{\prime} ; d^{\prime}, e^{\prime} ; 1\right)$, where $G$ is a gamma quotient, and the transformed parameters $\left(a^{\prime}, b^{\prime}, c^{\prime} ; d^{\prime}, e^{\prime}\right)$ and the arguments of $G$ depend linearly and homogeneously on $(a, b, c ; d, e)$. One of these expressions is ${ }_{3} F_{2}(a, b, c ; d, e ; 1)$ itself. The others can be extracted, with effort, from tables originally prepared by Whipple [Sla66, § 4.3]. Prudnikov et al. list only two [PBM90, § 7.4.4, Eqs. (1)-(2)].

If ${ }_{3} F_{2}(a, b, d ; d, e ; 1)$ is defined, i.e., if $\operatorname{Re}(d+e-a-b-c)>0$ and neither of $d, e$ is a nonpositive integer, it is not necessarily the case that each of the 10 distinct expressions for it is also defined, since $\left(a^{\prime}, b^{\prime}, c^{\prime} ; d^{\prime}, e^{\prime}\right)$ may fail to satisfy these conditions. Conversely, ${ }_{3} F_{2}\left(a^{\prime}, b^{\prime}, c^{\prime} ; d^{\prime}, e^{\prime} ; 1\right)$ may be defined even if ${ }_{3} F_{2}(a, b, c ; d, e ; 1)$ is divergent, in which case $G_{3} F_{2}\left(a^{\prime}, b^{\prime}, c^{\prime} ; d^{\prime}, e^{\prime} ; 1\right)$ may optionally be viewed as its formal sum: which is meaningful in the sense of analytic continuation.

Theorem 3.1. If a variety $\mathcal{V} \subset \mathbb{C}^{3} \times \mathbb{C}^{2}$ has the gamma quotient summation property, then for all 5 ! transformations $T \in \mathfrak{T} \subset G L(5, \mathbb{C})$, so does the linearly transformed variety $T \mathcal{V}$. That is, the property belongs to an entire $\mathfrak{T}$-orbit. 
Proof. This is immediate. A gamma quotient is associated to each $T$, but a product of gamma quotients is a gamma quotient.

When investigating the relation between Slater's formula and those of Dixon, Watson, and Whipple, one should look not merely at the intersection of the corresponding varieties, but also at the intersections of their images under all $T \in \mathfrak{T}$. An unexpected overlap could show up among the latter, even if the varieties themselves intersect properly.

It is well known that the Dixon, Watson, and Whipple 3-planes lie on a single T-orbit AAR99, Theorem 3.5.5]. In fact Whipple derived his formula (and the general case of Watson's formula) by applying appropriate $T \in \mathfrak{T}$ to Dixon's formula Whi24. An obvious question is whether there are other 3-planes on the orbit. There could be as many as 5 ! in all, but the upper bound shrinks to 10 if 3-planes related by permutations in $S_{3} \times S_{2}$ are identified. Whipple asserted without proof what amounts to the following.

Theorem 3.2. Up to separate permutations of upper and lower parameters, the Dixon-Watson-Whipple $\mathfrak{T}$-orbit in the space of 3-planes in $\mathbb{C}^{3} \times \mathbb{C}^{2}$ comprises the Dixon, Watson, and Whipple 3-planes, and no others.

Remark. A recent paper Ext99 produces a 3-plane different from any of these by applying a transformation in $\mathfrak{T}$ to Dixon's formula, but it unfortunately contains an algebra error. Correcting the error reveals that up to separate permutations, the 3 -plane is unchanged.

Proof of Theorem 3.2. The Dixon 3-plane is specified by $d=a-b+1$ and $e=$ $a-c+1$, or equivalently, $(2 y+z)+(2 u+v)=1$ and $(y+2 z)+(u+2 v)=1$. Due to the symmetrical role played by $y, z$ and $u, v$, its possible images under permutations of $x, y, z, u, v$ are bijective with the partitions of $x, y, z, u, v$ of type $2+2+1$. Up to separate permutations of $x, y, z$ and $u, v$, there are only three such partitions, namely $\{y, z\},\{u, v\},\{x\}$; and $\{y, z\},\{x, u\},\{v\}$; and $\{y, v\},\{x, u\},\{z\}$. By examination, these correspond to the 3-planes of (3.1)-(3.3).

Other 3-planes in $\mathbb{C}^{3} \times \mathbb{C}^{2}$ with the gamma quotient summation property are known, and when investigating the overlap between the $r=2$ case of Slater's formula and known results, one should consider them as well. No careful description of their behavior under Thomae's transformations seems to have been published. This gap is partially filled by the following theorem, which is proved similarly.

Theorem 3.3. For each integer $n \geqslant 1$, there is a $\mathfrak{T}$-orbit of 3 -planes in $\mathbb{C}^{3} \times \mathbb{C}^{2}$ with the gamma quotient summation property, which up to separate permutations of upper and lower parameters comprises exactly five 3-planes. Two correspond to

$$
{ }_{3} F_{2}\left(\begin{array}{c}
-n, a, b \\
c, 1+a+b-c-n
\end{array}\right)=\Gamma\left[\begin{array}{l}
c-a+n, c-b+n, c, c-a-b \\
c-a, c-b, c+n, c-a-b+n
\end{array}\right],
$$

$$
{ }_{3} F_{2}\left(\begin{array}{c}
a, b, c \\
a-n, 1+b
\end{array}\right)=\Gamma\left[\begin{array}{c}
1+b-a+n, 1-a, 1-c, 1+b \\
1+b-a, 1-a+n, 1+b-c
\end{array}\right] .
$$


In (i) and (ii) it is assumed that no lower parameter is a nonpositive integer, and in (ii), that the parametric excess has positive real part. The remaining 3-planes, three in number (up to separate permutations), consist of points that violate these constraints, so the corresponding identities are formal sums of divergent ${ }_{3} F_{2}(1)$ 's.

Remark. The identity (i) is the Pfaff-Saalschütz formula, which sums any terminating 1-balanced ${ }_{3} F_{2}(1)$. It and (ii) appear in Prudnikov et al. as Eqs. (88) and (15). To obtain the quotient on the right-hand side of (ii) in the above form, the reflection formula $\Gamma(z) \Gamma(1-z)=\pi \csc (\pi z)$ must be used repeatedly.

The remaining three identities, not given here, can be made sense of by analytic continuation in $\mathbb{C}^{3} \times \mathbb{C}^{2}(\mathrm{cf}$. Wim81, Wim83). The term 'Pfaff-Saalschütz orbit of index $n$ ' will refer to the T-orbit of the theorem, including the 'divergent' 3 -planes.

The Pfaff-Saalschütz orbits are not the only $\mathfrak{T}$-orbits of 3-planes, other than the Dixon-Watson-Whipple orbit, that have the gamma quotient summation property. An additional orbit arises from the summation formula

$$
{ }_{3} F_{2}\left(\begin{array}{c}
a, 1+\frac{1}{2} a, c \\
\frac{1}{2} a, e
\end{array}\right)=\Gamma\left[\begin{array}{c}
e, e-a-c-1, e+c-a \\
e-a, e-c, e+c-a-1
\end{array}\right] .
$$

This little-known identity is due to Shukla Shu58, who derived it in an indirect way. The special terminating case (i.e., $c=-n$ ) was earlier derived by Bailey, and is the only case listed in Prudnikov et al. PBM90, $§ 7.4 .4$, Eq. (106)]. Actually, (3.6) has a straightforward proof. The ${ }_{3} F_{2}(1)$ has an upper parameter that exceeds a lower by unity, so it can be written as a combination of two ${ }_{2} F_{1}(1)$ 's Luk75, $\S 5.2 .4]$, and evaluated. The 3-plane of (3.6) lies on its own T-orbit.

Yet another 3-parameter summation of ${ }_{3} F_{2}(1)$ in which the right side is a gamma quotient appears in Prudnikov et al. [PBM90, § 7.4.4], as Eq. (19). Unfortunately, study reveals that this formula, of unclear origin, is erroneous; it is valid only if the series terminates. So it does not give rise to a further $\mathfrak{T}$-orbit of 3 -planes.

Finally, the distinct Thomae-transformed Slater summation formulas can be enumerated. There are only three, due to the high degree of symmetry of Theorem 2.3 ,

Theorem 3.4. There is a T-orbit of 3 -dimensional algebraic varieties in $\mathbb{C}^{3} \times \mathbb{C}^{2}$ with the gamma quotient summation property, which up to separate permutations of upper and lower parameters comprises exactly three varieties, which are not 3planes. They correspond to the following quadratically constrained identities:

(i) If $a b+b c+c a=(d-1)(e-1)$ and $d+e-a-b-c=2$, then

$$
{ }_{3} F_{2}\left(\begin{array}{c}
a, b, c \\
d, e
\end{array}\right)=\Gamma\left[\begin{array}{c}
d, e \\
a+1, b+1, c+1
\end{array}\right] .
$$

(ii) If $(a-1)(b-1)=[(a-1)+(b-1)-(e-1)] c$, then

$$
{ }_{3} F_{2}\left(\begin{array}{c}
a, b, c \\
c+2, e
\end{array}\right)=\Gamma\left[\begin{array}{c}
e, e-a-b+2, c+2 \\
e-a+1, e-b+1, c+1
\end{array}\right],
$$

provided $\operatorname{Re}(e-a-b+2)>0$.

(iii) If $(a-1)(b-1)=(d-2)(e-2)$, then

$$
{ }_{3} F_{2}\left(\begin{array}{c}
a, b, 2 \\
d, e
\end{array}\right)=\frac{(d-1)(e-1)}{d+e-a-b-2},
$$

provided $\operatorname{Re}(d+e-a-b-2)>0$.

In each identity, it is assumed that no lower parameter is a nonpositive integer. 
Remark. Each of (i) (iii), if the quadratic constraint on its parameters is dropped, can be generalized to a formula in which a second ${ }_{3} F_{2}(1)$ appears on the right-hand side. For example, (i) generalizes to a nonterminating version of the SheppardAnderson identity, and (iii) to Eq. (27) of Prudnikov et al. [PBM90, § 7.4.4].

Numerical experimentation reveals that (ii) and (iii) hold whenever the series terminates, even if its parametric excess has a nonpositive real part. In this regard they differ from the formulas of Dixon, Watson, and Whipple. In the general terminating case, those three formulas must be modified Bai53, Dzh64].

If a lower parameter is a nonpositive integer and an upper one is a nonpositive integer of smaller magnitude, the series is usually regarded as terminating. Experimentation reveals that (iii) holds in this case, though (i) and (ii) do not.

Proof of Theorem 3.4. Formula (i) is a restatement of the $r=2$ case of Theorem 2.3. It follows from (3.5) that in terms of $x, y, z, u, v$, its variety is

$$
\left\{\begin{aligned}
x y+y z+z x+u^{2}+v^{2}+u v+u+v & =1 \\
x+y+z & =2 .
\end{aligned}\right.
$$

Due to the symmetrical role played by $x, y, z$ and $u, v$, up to separate permutations there are only three images of this variety under a permutation $\sigma \in S_{5}$. It suffices to consider the permutations $x, y, z, u, v$; and $x, y, u, z, v$; and $x, v, u, z, y$. By examination, these yield (i) (iii), respectively. Interestingly, the gamma quotient in (iii) reduces to a rational function of the parameters.

Just as the $r=2$ case of Theorem 2.3 gives rise to the $\mathfrak{T}$-orbit of Theorem 3.4 the $r=2$ case of Theorem 2.4 gives rise to a $\mathfrak{T}$-orbit of 3-dimensional algebraic varieties. However, the three ${ }_{3} F_{2}(1)$ summations on that orbit are not of gamma quotient type. It is clear from the right side of Theorem 2.4 that each yields not a gamma quotient, but a gamma quotient times a rational function of the hypergeometric parameters.

The extent to which the identities of Theorem 3.4 and those of Dixon, Watson, Whipple and Pfaff-Saalschütz overlap one another will now be considered. The term 'Slater orbit' will refer to the T-orbit of Theorem 3.4

Definition. If $\mathfrak{V}$ and $\mathfrak{W}$ are two $\mathfrak{T}$-orbits of algebraic varieties in $\mathbb{C}^{3} \times \mathbb{C}^{2}$ (satisfying $m+n \geqslant 5$, where $m, n$ are the dimensions of the varieties in $\mathfrak{V}, \mathfrak{W}$, respectively), they are said to intersect improperly iff there is at least one $\mathcal{V} \in \mathfrak{V}$ and one $\mathcal{W} \in \mathfrak{W}$ that intersect improperly. By invariance under $\mathfrak{T}$, this occurs iff for all $\mathcal{V} \in \mathfrak{V}$, there is at least one $\mathcal{W} \in \mathfrak{W}$ such that $\mathcal{V}$ and $\mathcal{W}$ intersect improperly.

\section{Theorem 3.5.}

(i) The Slater orbit and Dixon-Watson-Whipple orbit intersect improperly.

(ii) For all $n \geqslant 1$, the Slater orbit and the Pfaff-Saalschütz orbit of index $n$ do not intersect improperly.

(iii) For all $n \geqslant 1$, the Dixon-Watson-Whipple orbit and the Pfaff-Saalschütz orbit of index $n$ do not intersect improperly.

Proof. (i), It suffices to show that the parametric restrictions of Theorem 3.4(i) and Dixon's formula, taken together, result in there being two free parameters rather 
than one. By examination, the 2-parameter formula

$$
{ }_{3} F_{2}\left(\begin{array}{c}
2 b+2 c, b, c \\
1+b+2 c, 1+2 b+c
\end{array}\right)=\Gamma\left[\begin{array}{c}
1+b+2 c, 1+2 b+c \\
1+2 b+2 c, 1+b, 1+c
\end{array}\right],
$$

the variety corresponding to which is a 2-plane, is a specialization of both Dixon's formula and Theorem 3.4(i) so their overlap is improper.

[ii) It suffices to compare Theorem 3.3](i) or Theorem 3.3](ii) with each of Theorem [3.4](i) [(ii)](iii), and verify that their parametric constraints, taken together, result in there being a single free parameter. (Each comparison is really 3 ! 2! separate ones, since all separate permutations of upper and lower parameters must be considered.) It is easier to use Theorem 3.3](i), Its series is 1-balanced and that of Theorem 3.4(i) is 2-balanced, so the corresponding varieties do not intersect; and only Theorem [3.4](ii), (iii) need to be compared with. Details are left to the reader.

(iii). This is similar to the proof of (ii). It is easy to compare Theorem[3.3](ii) with each of (3.1) - (3.3), and verify that each possible overlap (separate permutations of upper and lower parameters being allowed) has only one free parameter.

It follows from Theorem 3.5(i) that each of the Dixon, Watson, and Whipple 3-planes improperly intersects at least one of the three algebraic varieties of Theorem 3.4. Conversely, each of those algebraic varieties improperly intersects at least one of the Dixon, Watson, and Whipple 3-planes. In these statements the phrase "up to separate permutations of upper and lower parameters" is understood.

The following examples may be instructive. Up to separate permutations,

$$
{ }_{3} F_{2}\left(\begin{array}{c}
2, b, c \\
\frac{3}{2}+\frac{1}{2} b, 2 c
\end{array}\right)=\frac{(1+b)(1-2 c)}{(1+b-2 c)}
$$

is a specialization of both Watson's formula and Theorem 3.4(iii) Similarly,

$$
{ }_{3} F_{2}\left(\begin{array}{c}
a, 1-a, c \\
2+a, 2 c-a-1
\end{array}\right)=\Gamma\left[\begin{array}{c}
2 c-a-1, c, 2+a \\
c-a, 2 c-1,1+a
\end{array}\right]
$$

is a specialization of both Whipple's formula and Theorem 3.4 (ii) Additional examples of 2-parameter overlap can be constructed. In $\mathbb{C}^{3} \times \mathbb{C}^{2}$, the Slater variety and its images under Thomae's transformations intersect the Dixon-Watson-Whipple 3 -planes quite extensively.

The preceding results, such as (3.7)-(3.9), clarify a remark of Slater Sla66, $\S 2.6 .1]$ that the $r=2$ case of her summation formula is "a disguised form of Dixon's theorem". It is better described as an extension of a 2-parameter special case of Dixon's theorem, and if Thomae's transformations are taken into account, the unexpected degree of overlap between the two summation formulas becomes an unexpected degree of overlap between two $\mathfrak{T}$-orbits.

\section{4. ${ }_{2} F_{1}(-1)$ SUMmations}

If $\operatorname{Re}(c-a-b)>-1$, then ${ }_{2} F_{1}(a, b ; c ;-1)$ is convergent, though in general it cannot be evaluated in terms of the gamma function. In the well-poised case, it can be evaluated as a single gamma quotient. Kummer's theorem states that

$$
{ }_{2} F_{1}\left(\begin{array}{c|c}
a, b & -1 \\
1+a-b & -1
\end{array}\right) \Gamma\left[\begin{array}{c}
1+a-b, 1+\frac{1}{2} a \\
1+a, 1+\frac{1}{2} a-b
\end{array}\right],
$$


provided $1+a-b$ and $1+\frac{1}{2} a$ are not nonpositive integers. Prudnikov et al. PBM90, $\S 7.3 .6]$ supply a list of known evaluations of ${ }_{2} F_{1}(-1)$ with one or more free parameters. Besides Kummer's formula, these include several multi-term evaluations contiguous to it, in the sense that they follow by contiguous function relations.

Kummer's formula is often viewed as a consequence of a quadratic transformation on the ${ }_{2} F_{1}$ level, based on $x \mapsto R(x)=-4 x /(1-x)^{2}$, which applies to well-poised series AAR99, $\S 3.1$ ]. Since $R(-1)=1$, the transformation permits the summation of well-poised ${ }_{2} F_{1}(-1)$ series by Gauss's formula, leading to (4.1). Kummer's formula will be viewed differently here. Whipple Whi29 showed that

$$
{ }_{2} F_{1}\left(\begin{array}{c}
2 \alpha, \beta \\
2 \gamma-\beta
\end{array} \mid-1\right)=\Gamma\left[\begin{array}{c}
2 \gamma-2 \alpha, 2 \gamma-\beta \\
2 \gamma-2 \alpha-\beta, 2 \gamma
\end{array}\right]{ }_{3} F_{2}\left(\begin{array}{c}
\alpha, \frac{1}{2}+\alpha, \beta \\
\gamma, \frac{1}{2}+\gamma
\end{array}\right),
$$

provided that both sides are defined. So there is a 3-plane in the $\mathbb{C}^{3} \times \mathbb{C}^{2}$ parameter space, i.e., $\mathcal{W}_{0}=\left\{\left(\alpha_{1}, \alpha_{2}, \alpha_{3} ; \beta_{1}, \beta_{2}\right) \mid \alpha_{2}=\alpha_{1}+\frac{1}{2}, \beta_{2}=\beta_{1}+\frac{1}{2}\right\}$, on which any evaluation of ${ }_{3} F_{2}(1)$ leads to an evaluation of a ${ }_{2} F_{1}(-1)$. By covariance under the Thomae group $\mathfrak{T}$, the same is true of all 3-planes of the form $T \mathcal{W}_{0}, T \in \mathfrak{T}$. Whipple showed that up to separate permutations of upper and lower parameters, there are exactly six such 3 -planes, including $\mathcal{W}_{0}$ itself, and the term 'Whipple ${ }_{2} F_{1}(-1)$ orbit' will refer to this $\mathfrak{T}$-orbit. His paper contains five additional Thomae-transformed versions of the relation (4.2), which will not be needed explicitly.

If any $\mathfrak{T}$-orbit of algebraic varieties in $\mathbb{C}^{3} \times \mathbb{C}^{2}$ has the gamma quotient summation property, its intersection with the Whipple ${ }_{2} F_{1}(-1)$ orbit will too, if nonempty, and one or more evaluations of ${ }_{2} F_{1}(-1)$ as a single gamma quotient will result. In the framework of this paper, this is the origin of (4.1). Kummer's formula arises from the nonempty intersection of the Dixon-Watson-Whipple orbit with the Whipple ${ }_{2} F_{1}(-1)$ orbit. In particular, it arises from the intersection of a Dixon 3-plane with the Whipple ${ }_{2} F_{1}(-1) 3$-plane, which produces a 2 -plane rather than a line (and is therefore improper). Combining (3.1) with (4.2) yields the 2-parameter formula

$$
\begin{aligned}
{ }_{2} F_{1}\left(\begin{array}{c}
a, b \\
1+a-b
\end{array}\right. & -1) \\
= & \Gamma\left[\begin{array}{c}
1+2 a-2 b, 1+a-b \\
1+a-2 b, 1+2 a-b
\end{array}\right]{ }_{3} F_{2}\left(\begin{array}{c}
\frac{1}{2} b, \frac{1}{2}+\frac{1}{2} b, a \\
\frac{1}{2}+a-\frac{1}{2} b, 1+a-\frac{1}{2} b
\end{array}\right) \\
= & \Gamma\left[\begin{array}{c}
1+2 a-2 b, 1+a-b \\
1+a-2 b, 1+2 a-b
\end{array}\right] \\
& \times \Gamma\left[\begin{array}{c}
1+\frac{1}{2} a, \frac{1}{2}+\frac{1}{2} a-b, 1+a-\frac{1}{2} b, \frac{1}{2}+a-\frac{1}{2} b \\
1+a, \frac{1}{2}+a-b, 1+\frac{1}{2} a-\frac{1}{2} b, \frac{1}{2}+\frac{1}{2} a-\frac{1}{2} b
\end{array}\right] .
\end{aligned}
$$

If this is simplified with the aid of the duplication formula for the gamma function, it becomes Kummer's formula.

In the same way, many ${ }_{2} F_{1}(-1)$ summations can be derived by intersecting the Pfaff-Saalschütz and Slater orbits with the Whipple ${ }_{2} F_{1}(-1)$ orbit. Each intersection turns out to be proper, producing a union of lines, or (in the Slater case) algebraic curves. The 1-parameter summation formulas corresponding to the latter, with nonlinear parametric constraints, are quite exotic. They are given in Theorem 4.2 and (4.5)-4.6) below.

Actually, there are intersections between the Dixon-Watson-Whipple and Whipple ${ }_{2} F_{1}(-1)$ orbits other than the improper one yielding Kummer's formula. Surprisingly, the resulting 1-parameter identities have never been systematically worked 
out. Theorem 4.1 does this. It refers to Euler's transformation in the form

$$
\begin{aligned}
& { }_{2} F_{1}\left(\begin{array}{c|c}
a, b & -1 \\
c &
\end{array}\right)=\Gamma\left[\begin{array}{c}
\frac{1}{2}, 1+c-a-b \\
\frac{1}{2}+\frac{1}{2} c-\frac{1}{2} a-\frac{1}{2} b, 1+\frac{1}{2} c-\frac{1}{2} a-\frac{1}{2} b
\end{array}\right] \\
& \times{ }_{2} F_{1}\left(\begin{array}{c|c}
c-a, c-b & -1 \\
c & -
\end{array}\right)
\end{aligned}
$$

which follows from (1.1) by the duplication formula, and leaves the gamma quotient summation property for ${ }_{2} F_{1}(-1)$ unaffected. Note that in (4.3) the parametric excess $s$ of each series is the negative of that of the other. Since each series, if nonterminating, converges only if $\operatorname{Re} s>-1$, it is possible for one to be convergent and the other divergent. If so, (4.3) will supply a formal sum for the divergent one.

The reason why (4.3) is relevant here is the following. The Whipple ${ }_{2} F_{1}(-1)$ orbit comprises all 3-planes of the form $T \mathcal{W}_{0}, T \in \mathfrak{T}$. Whipple's identity (4.2) supplies a canonical map $P$ of $\mathcal{W}_{0}$ onto $\mathbb{C}^{2} \times \mathbb{C}^{1}$, the parameter space of ${ }_{2} F_{1}(-1)$. Any 3-plane $\mathcal{W}=T \mathcal{W}_{0}$ is projected onto $\mathbb{C}^{2} \times \mathbb{C}^{1}$ by $P T^{-1}$. This map is not unique, since there are Thomae transformations $T$ other than the identity that map $\mathcal{W}_{0}$ onto itself, yet have the property that $P T^{-1} \neq P$. But as Whipple noted in somewhat different language Whi29, the map from each $\mathcal{W}$ onto $\mathbb{C}^{2} \times \mathbb{C}^{1}$ is unique up to composition with a subsequent map $(a, b ; c) \mapsto(c-a, c-b ; c)$, i.e., up to a subsequent Euler transformation.

Theorem 4.1. Up to separate permutations of upper and lower parameters and up to Euler's transformation, the intersection of the 3-planes on the Dixon-WatsonWhipple $\mathfrak{T}$-orbit and the 3 -planes on the Whipple ${ }_{2} F_{1}(-1) \mathfrak{T}$-orbit, when projected to $\mathbb{C}^{2} \times \mathbb{C}^{1}$ via the map in Whipple's identity (4.2), comprises (a) the Kummer 2-plane, and (b) exactly four lines. The latter correspond to the following:

(i)

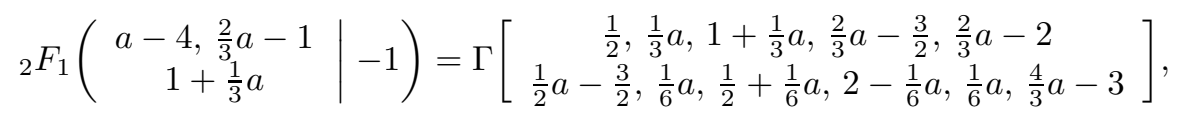

(ii)

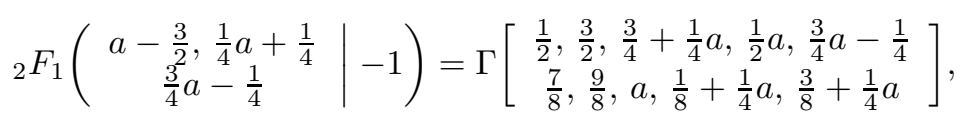

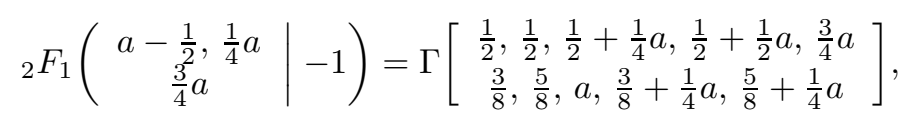

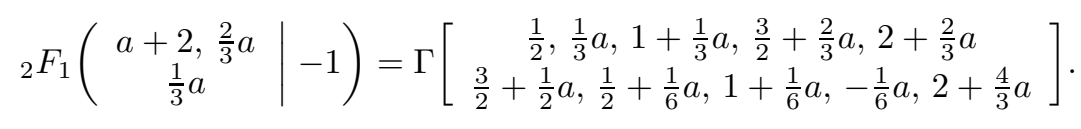

In each of the above it is assumed that the parametric excess of the ${ }_{2} F_{1}(-1)$ series has real part greater than -1 , with no lower parameter of the series or upper parameter of the gamma quotient equal to a nonpositive integer. 
Remark. Each of (i) (iv) is written as a ${ }_{2} F_{1}(a+\nu, b ; a-b ;-1)$ evaluation, to agree with the convention of Vidūnas [Vid02]. Respectively, $\nu$ equals $-4,-\frac{3}{2},-\frac{1}{2}, 2$. In each, $b$ is some linear function of $a$. The quantity $\nu$ measures the nearness of the series to well-poisedness. When $\nu=-1$, the series is well-poised and can be summed by Kummer's formula, with no restriction on $b$, to give the gamma quotient $\Gamma(a-b) \Gamma\left(\frac{1}{2}+\frac{1}{2} a\right) / \Gamma(a) \Gamma\left(\frac{1}{2}+\frac{1}{2} a-b\right)$.

Note that by using the duplication formula, the right side of (i) can be written in a simpler way, as $(3 / 4) \Gamma\left(1+\frac{1}{3} a\right) \Gamma\left(\frac{1}{2} a-1\right) / \Gamma(a-2) \Gamma\left(2-\frac{1}{6} a\right)$. The right sides of (ii) (iv) can also be rewritten in various ways.

Proof of Theorem 4.1. This is straightforward though tedious. It suffices to compare the Whipple ${ }_{2} F_{1}(-1)$ formula (4.2) with each of (3.1)-(3.3), and determine the possible ways in which their respective parametric constraints on ${ }_{3} F_{2}(1)$ can be simultaneously satisfied. (Each of these three comparisons is really 3 ! 2 ! separate comparisons.) Every success yields a nonempty intersection of the corresponding 3 -planes, and a parametrized gamma quotient evaluation of ${ }_{2} F_{1}(-1)$.

An example is the following. Specializing parameters in (4.2) yields

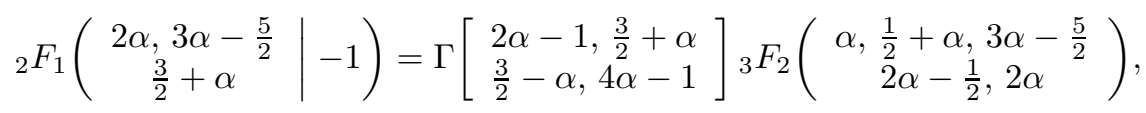

in which the ${ }_{3} F_{2}(1)$ may be evaluated by (3.2), Watson's formula. Performing the evaluation and changing the independent variable from $\alpha$ to $a=\frac{3}{2}-3 \alpha$ yields the 1-parameter evaluation of ${ }_{2} F_{1}(-1)$ shown in Theorem 4.1](i).

Besides the 2-parameter formula of Kummer, the comparisons yield eight 1parameter formulas in all, but these split into four pairs, each being related by (4.3), Euler's transformation. Theorem 4.1 lists only a single member of each.

Theorem 4.1 is closely related to the results of Vidūnas, who recently worked out a systematic way of generating ${ }_{2} F_{1}(-1)$ evaluations contiguous to Kummer's formula Vid02. He showed that for all integer $\nu$,

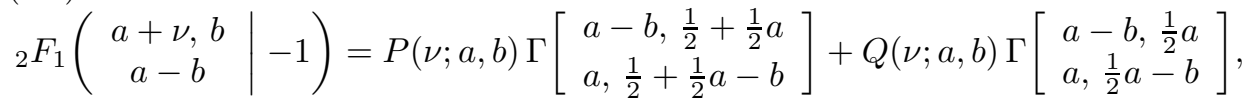

where $P(\nu ; a, b)$ and $Q(\nu ; a, b)$ are rational in $a, b$. For any $\nu \in \mathbb{Z}$, setting $P(\nu ; a, b)$ or $Q(\nu ; a, b)$ to zero constrains $a, b$ algebraically, and if the constraint is satisfied, the remaining term on the right-hand side of (4.4) becomes (almost) a gamma quotient summation of ${ }_{2} F_{1}(-1)$. If $Q(\nu ; a, b)$ or $P(\nu ; a, b)$, respectively, factors into quotients of linear polynomials, then it will be, in fact, a gamma quotient.

Vidūnas noticed that if $\nu=-4$, then $b$ is constrained to be a linear function of $a$, and he worked out a ${ }_{2} F_{1}(-1)$ summation equivalent to Theorem 4.1](i). When $\nu=2$, his approach also yields a gamma quotient summation, equivalent to Theorem 4.11(iv)] A nice feature of his treatment is that it makes clear where the reflection symmetry through $\nu=-1$ comes from. (The set $\left\{-4,-\frac{3}{2},-\frac{1}{2}, 2\right\}$ is invariant under this operation.) He showed that if $\nu \in \mathbb{Z}$, reflecting $P(\nu ; a, b)$, $Q(\nu ; a, b)$ through $\nu=-1$ simply multiplies them by gamma quotients. However, his results do not immediately extend to the noninteger $\nu$.

Finally, the exotic ${ }_{2} F_{1}(-1)$ summations arising from Slater's formula can be given. 
Theorem 4.2. Up to separate permutations of upper and lower parameters and up to Euler's transformation, the intersection of the 3-dimensional algebraic varieties on the Slater $\mathfrak{T}$-orbit and the 3 -planes on the Whipple ${ }_{2} F_{1}(-1) \mathfrak{T}$-orbit, when projected to $\mathbb{C}^{2} \times \mathbb{C}^{1}$ via the map in Whipple's identity (4.2), comprises (a) three lines that lie in the Kummer 2-plane, (b) one line that lies in the displaced 2-plane with $\nu=-4$, and (c) two algebraic curves other than lines. The last correspond to the following identities, with parameters constrained both quadratically and linearly:

(i) If $2(\alpha-\beta)^{2}-3 \alpha+4 \beta+1=0$, then

$$
{ }_{2} F_{1}\left(\begin{array}{c|c}
\beta-2 \alpha+3,3 \\
\beta+3
\end{array} \mid-1\right)=\frac{1}{4}\left[\frac{(\beta+1)(\beta+2)}{2 \beta-2 \alpha+3}\right] .
$$

(ii) If $2(\alpha-\beta)^{2}-3 \alpha+6 \beta+1=0$, then

$$
{ }_{2} F_{1}\left(\begin{array}{c|c}
\beta-2 \alpha+4,4 & -1 \\
\beta+4 &
\end{array}\right)=\frac{1}{4}\left[\frac{(\beta+1)(\beta+2)(\beta+3)}{(2 \beta-2 \alpha+4)(2 \beta-2 \alpha+5)}\right] .
$$

In both (i) and (ii) it is assumed that the ${ }_{2} F_{1}(-1)$ series converges.

Proof. As in the proof of Theorem 4.1, this is straightforward but lengthy. It suffices to compare the Whipple ${ }_{2} F_{1}(-1)$ formula (4.2) with each of Theorem 3.4(i) (iii), and determine the ways in which their respective parametric constraints on ${ }_{3} \bar{F}_{2}(1)$ can be simultaneously satisfied. (Each comparison is really $3 ! 2$ ! separate ones.) Every success yields a nonempty intersection of a Whipple ${ }_{2} F_{1}(-1) 3$-plane with a 3-dimensional variety on the Slater orbit, and a parametrized gamma quotient evaluation of ${ }_{2} F_{1}(-1)$. Each intersection turns out to be proper, i.e., 1-dimensional.

An example is the following. Specializing parameters in (4.2) yields

$$
{ }_{2} F_{1}\left(\begin{array}{c|c}
2 \alpha, \beta \\
3+\beta
\end{array} \mid-1\right)=\Gamma\left[\begin{array}{c}
3+2 \beta-2 \alpha, 3+\beta \\
3+\beta-2 \alpha, 3+2 \beta
\end{array}\right]{ }_{3} F_{2}\left(\begin{array}{c}
\alpha, \frac{1}{2}+\alpha, \beta \\
\frac{3}{2}+\beta, 2+\beta
\end{array}\right) .
$$

If $2(\alpha-\beta)^{2}-3 \alpha+4 \beta+1=0$, the ${ }_{3} F_{2}(1)$ may be evaluated by Theorem 3.4)(ii). Performing the evaluation, and also applying Euler's transformation in the form (4.3) to the ${ }_{2} F_{1}(-1)$ on the left-hand side, yields the 1-parameter formula for ${ }_{2} F_{1}(-1)$ shown in Theorem 4.2(i).

By uniformizing the two algebraic curves of the theorem, i.e., parametrizing them by an auxiliary variable $t \in \mathbb{C}$, the identities (i) and (ii) of the theorem can be converted to the mysterious parametric forms

$$
\begin{aligned}
{ }_{2} F_{1}\left(\begin{array}{c}
2 t^{2}-5 t+4,3 \\
-2 t^{2}+3 t+2
\end{array} \mid-1\right) & =-\frac{1}{4} t\left(2 t^{2}-3 t-1\right), \\
{ }_{2} F_{1}\left(\begin{array}{c}
6 t^{2}-11 t+6,4 \\
-6 t^{2}+5 t+3
\end{array} \mid-1\right) & =-\frac{1}{24} t(6 t+1)\left(6 t^{2}-5 t-2\right) .
\end{aligned}
$$

Each holds for all $t \in \mathbb{C}$ for which the ${ }_{2} F_{1}(-1)$ series converges.

The identities (4.5)-(4.6) could alternatively be derived from Gauss's three-term contiguous function relations for ${ }_{2} F_{1}$, mentioned in Section 2. In fact, iterating the relation

$$
\begin{array}{r}
(c-b){ }_{2} F_{1}\left(\begin{array}{c|c}
a, b-1 & x \\
c & x
\end{array}\right)+(2 b-c-b x+a x){ }_{2} F_{1}\left(\begin{array}{c|c}
a, b & x \\
c & x
\end{array}\right. \\
+b(x-1){ }_{2} F_{1}\left(\begin{array}{c}
a, b+1 \\
c
\end{array} \mid x\right)=0
\end{array}
$$


so as to express ${ }_{2} F_{1}(a, b ; c ; x)$ with $b=2,3$ as a combination of ${ }_{2} F_{1}(a, 0 ; c ; x) \equiv 1$ and ${ }_{2} F_{1}(a, 1 ; c ; x)$, and setting the coefficient of ${ }_{2} F_{1}(a, 1 ; c ; x)$ to zero and uniformizing the resulting $x$-dependent algebraic curve in $\mathbb{C}^{2} \ni(a, c)$, yields the identities

$$
\begin{gathered}
{ }_{2} F_{1}\left(\begin{array}{c|c}
-x^{-1} t+\left(1-x^{-1}\right), 2 \\
-t+1
\end{array} \mid x\right)=\frac{t}{x-1}, \\
{ }_{2} F_{1}\left(\begin{array}{c}
\left.\left(1-x^{-1}\right) t^{2}+\left(-2+3 x^{-1}\right) t+\left(2-2 x^{-1}\right), 3 \mid x\right) \\
(x-1) t^{2}+(-x+2) t+2
\end{array}\right. \\
=\frac{t\left[(-x+1) t^{2}+(x-2) t-1\right]}{2(x-1)},
\end{gathered}
$$

the latter of which subsumes (4.5). This sequence of identities cannot be continued indefinitely, since the $b=4$ curve is cubic: it is the zero-set of a cubic polynomial in $(a, c)$, with coefficients polynomial in $x$. The genus of an irreducible algebraic curve of degree $n$ is $\left(\begin{array}{c}n-1 \\ 2\end{array}\right)$ minus the number of its double points, which is generically zero. So one would expect that generically, i.e., except at isolated values of $x$, this cubic curve would be irreducible of genus 1 , ruling out a uniformization by rational functions. Some computation reveals that the situation is not so bad as that: irrespective of $x$, the $b=4$ curve always has genus zero. In fact, if $x$ is one of $-1,1 / 2,2$, then it turns out to be reducible: the union of a quadratic curve and a line. When $x=-1$, the quadratic component can be uniformized by polynomials, yielding (4.6). However, the problem of positive genus does set in when $b \geqslant 5$. Fuller details of this alternative approach will appear elsewhere.

Actually, the first proof of (4.5)-(4.6), which was based not on contiguous function relations but rather on Theorem 4.2, and therefore on the generalized Euler transformation Theorem 2.1, has much to recommend it. Its existence suggests that Theorem 2.1 encapsulates much of the power of the contiguous function relations for ${ }_{r+1} F_{r}$ to generate one-term summation formulas.

\section{REFERENCES}

[AAR99] G. E. Andrews, R. Askey, and R. Roy, Special Functions, Encyclopedia of Mathematics and Its Applications, vol. 71, Cambridge University Press, Cambridge, UK, 1999. MR1688958 (2000g:33001)

[Ask89] R. Askey, Variants of Clausen's formula for the square of a special ${ }_{2} F_{1}$, in Number Theory and Related Topics, Tata Institute of Fundamental Research Studies in Mathematics, no. 12, Oxford University Press, Oxford, 1989, pp. 1-12. MR.1441321|(98f:33003)

[Ask94] _ A look at the Bateman project, in The Mathematical Legacy of Wilhelm Magnus: Groups, Geometry, and Special Functions (W. Abikoff, J. S. Birman, and K. Kuiken, eds.), Contemporary Mathematics, vol. 169, American Mathematical Society, Providence, RI, 1994, pp. 29-43. MR1292896 (97f:33001)

[Bai53] W. N. Bailey, On the sum of a terminating ${ }_{3} F_{2}$, Quart. J. Math. Oxford Ser. (2) 4 (1953), no. 15, 237-240. MR0057381 (15:218d)

[BLS87] W. A. Beyer, J. D. Louck, and P. R. Stein, Group theoretical basis of some identities for the generalized hypergeometric function, J. Math. Phys. 28 (1987), no. 3, 497-508. MR0877220(88j:33003)

[Dzh64] V. A. Dzhrbashyan [Džrbašjan], On a theorem of Whipple, U.S.S.R. Comput. Math. and Math. Phys. 4 (1964), 190-194. MR0160945 (28:4154)

[Ext99] H. Exton, $A$ new two-term relation for the ${ }_{3} F_{2}$ hypergeometric function of unit argument, J. Comp. Appl. Math. 106 (1999), no. 2, 395-397. MR.1696419 (2000c:33006) 
[Gos76] R. Wm. Gosper, Jr., A calculus of series rearrangements, Algorithms and Complexity: New Directions and Recent Results (J. F. Traub and H. T. Kung, eds.), Academic Press, New York, 1976, pp. 121-151. MR0451617 (56:9899)

[Gou81] É. Goursat, Sur l'équation différentielle linéaire qui admet pour intégrale la série hypergéométrique, Ann. Sci. École Normale Sup. (2) 10 (1881), S3-S142.

[Gou72] H. W. Gould, Combinatorial identities: A standardized set of tables listing 500 binomial coefficient summations, Privately printed, Morgantown, WV, 1972. MR0354401 (50:6879)

[GS82] I. Gessel and D. Stanton, Strange evaluations of hypergeometric series, SIAM J. Math. Anal. 13 (1982), no. 2, 295-308. MR0647127 (83c:33002)

[Har23] G. H. Hardy, A chapter from Ramanujan's note-book, Proc. Cambridge Philos. Soc. 21 (1923), no. 5, 492-503.

[Koe98] W. Koepf, Hypergeometric Summation: An Algorithmic Approach to Summation and Special Function Identities, Vieweg-Verlag, Braunschweig, Germany, 1998. MR.1644447 (2000c:33002)

[KS03] C. Krattenthaler and K. Srinivasa Rao, Automatic generation of hypergeometric identities by the beta integral method, J. Comp. Appl. Math. 160 (2003), no. 1-2, 159-173. MR2022609 (2005a:33023)

[Luk69] Y. L. Luke, The Special Functions and Their Approximations, Academic Press, New York, 1969. MR0241700(39:3039) MR0249668(40:2909)

[Luk75] Mathematical Functions and Their Approximations, Academic Press, New York, 1975. MR0501762 (58:19039)

[Nib53] J. D. Niblett, Some hypergeometric identities, Pacific J. Math. 2 (1953), no. 2, 219-225. MR0047837 (13:940c)

[PBM90] A. P. Prudnikov, Iu. A. Brychkov, and O. I. Marichev, More Special Functions, Integrals and Series, vol. 3, Gordon and Breach, New York, 1990. MR 1054647 (91c:33001)

[PWZ96] M. Petkovšek, H. S. Wilf, and D. Zeilberger, $A=B$, A. K. Peters, Wellesley, MA, 1996. MR.1379802 (97j:05001)

[Rai45] E. D. Rainville, The contiguous function relations for ${ }_{p} F_{q}$ with applications to Bateman's $J_{n}^{u, v}$ and Rice's $H_{n}(\xi, p, v)$, Bull. Amer. Math. Soc. 51 (1945), 714-723. MR $0012726(7: 65 \mathrm{~d})$

[Roy87] R. Roy, Binomial identities and hypergeometric series, Amer. Math. Monthly 94 (1987), no. 1, 36-46. MR0873603 (88f:05012)

[Sea09] J. H. C. Searle, The summation of certain series, Messenger Math. 38 (1909), 138-144.

[Shu58] H. S. Shukla, Certain transformations of nearly-poised bilateral hypergeometric series of special type, Canad. J. Math. 10 (1958), no. 2, 195-201. MR0095298 (20:1801)

[Sla55] L. J. Slater, A note on the partial sum of a certain hypergeometric series, Math. Gaz. 39 (1955), 217-218.

[Sla66] - Generalized Hypergeometric Functions, Cambridge University Press, Cambridge, UK, 1966. MR0201688 (34:1570)

[Sta98] D. Stanton, A hypergeometric hierarchy for the Andrews evaluations, Ramanujan J. 2 (1998), no. 4, 499-509. MR 1665324 (99k:33015)

[Tho79] J. Thomae, Ueber die Funktionen, welche durch Reihen von der Form dargestellt werden $1+\frac{p}{1} \frac{p^{\prime}}{q^{\prime}} \frac{p^{\prime \prime}}{q^{\prime \prime}}+\frac{p}{1} \frac{p+1}{2} \frac{p^{\prime}}{q^{\prime}} \frac{p^{\prime}+1}{q^{\prime}+1} \frac{p^{\prime \prime}}{q^{\prime \prime}} \frac{p^{\prime \prime}+1}{q^{\prime \prime}+1}+\cdots$, J. Reine Angew. Math. 87 (1879), 26-73.

[Vid02] R. Vidūnas, A generalization of Kummer's identity, Rocky Mountain J. Math. 32 (2002), no. 2, 919-936. MR 1934920(2003j:33011)

[Vid04] - Algebraic transformations of Gauss hypergeometric functions, Preprint, available as arXiv:math.CA/0408269, 2004.

[Whi24] F. J. W. Whipple, A group of generalized hypergeometric series: Relations between 120 allied series of the type $F\left[\begin{array}{c}a, b, c \\ e, f\end{array}\right]$, Proc. London Math. Soc. (2) 23 (1924), no. 2, 104-114.

[Whi29] , On series allied to the hypergeometric series with argument -1 , Proc. London Math. Soc. (2) 30 (1929), no. 2, 81-94.

[Wim81] Jet Wimp, The computation of ${ }_{3} F_{2}(1)$, Internat. J. Comput. Math. 10 (1981), no. 1, 55-62. MR.0644716 (83d:65053)

[Wim83] - Irreducible recurrences and representation theorems for ${ }_{3} F_{2}$, Comput. Math. Appl. 9 (1983), no. 5, 669-678. MR0726815 (85b:33005) 
[Wim98] - The umbral calculus and identities for hypergeometric functions with special arguments, in Mathematical Essays in Honor of Gian-Carlo Rota (B. E. Sagan and R. P. Stanley, eds.), Progress in Mathematics, vol. 161, Birkhäuser, Boston/Basel, 1998, pp. 439-457. MR 1627394 (99i:05023)

[Zei92] D. Zeilberger, Gauss's ${ }_{2} F_{1}(1)$ cannot be generalized to ${ }_{2} F_{1}(x)$, J. Comp. Appl. Math. 39 (1992), no. 3, 379-382. MR1164298 (93i:33002)

Departments of Mathematics and Physics, University of Arizona, Tucson, Arizona 85721

E-mail address: rsm@math.arizona.edu

$U R L:$ http://www.math.arizona.edu/ rsm 